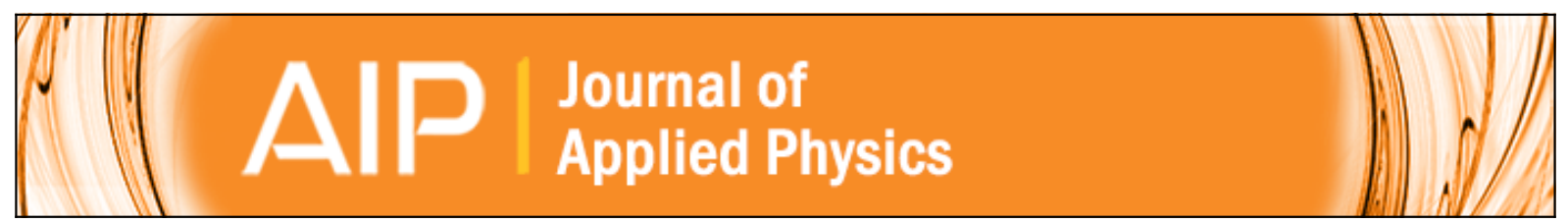

\title{
Implanted boron depth profiles in the AZ111 photoresist
}

R. B. Guimarães, L. Amaral, M. Behar, P. F. P. Fichtner, F. C. Zawislak, and D. Fink

Citation: Journal of Applied Physics 63, 2083 (1988); doi: 10.1063/1.341112

View online: http://dx.doi.org/10.1063/1.341112

View Table of Contents: http://scitation.aip.org/content/aip/journal/jap/63/6?ver=pdfcov

Published by the AIP Publishing

\section{$\underset{\substack{\text { Probusturg } \\ \text { AlP }}}{A}$ Re-register for Table of Content Alerts}

\section{Create a profile.}




\title{
Implanted boron depth proflles in the AZ 11 photoresist
}

\author{
R. B. Guimarães, L. Amaral, M. Behar, F.F.P. Fichtner, and F. C. Zawislak \\ Instituto de Fisica, Universidade Federal do Rio Grande do Sul, 90049 Porto Alegre, Brazil \\ D. Fink \\ Hahn-Meitner-Institut, Glienickerstrasse 100, D-1000 Perlin 39, West Germany
}

(Received 8 September 1987; accepted for publication 4 November 1987)

\begin{abstract}
The isotope ${ }^{10} \mathrm{~B}$ has been implanted into the photoresist $\mathrm{AZ111}$ in the $30-150 \mathrm{keV}$ energy range. The corresponding depth profiles have been analyzed using the ${ }^{10} \mathrm{~B}(n, \alpha)^{7} \mathrm{Li}$ reaction. At $60 \mathrm{keV}$, the profile changes from a regular shape to one with an additional tail directed towards the surface. Despite the nonregular shape of the ion distributions, it is possible to extract the characteristic range parameters such as projected range $R_{p}$, most probable range $\hat{R}$, and full width at half-maximum. Good agreement is found between the experimental results and the calculations by Ziegler, Biersack, and Littmark (ZBL). It is also shown that the tail distribution follows closely the $Z B L$ calculated ionization profles. A tentative explanation of this behavior is given.
\end{abstract}

\section{INTRODUCTIOA}

In recent years there has been a growing interest in ion irrasiation and/or implantation of polymers, essentially for the application in advanced microelectronic technology. Thin photoresist films are required in order to limit the area of microelectronic devices in which dopants are implanted. Projected ranges $\left(R_{p}\right)$ and range stragglings $\left(\Delta R_{p}\right)$ of the implanted ions must be known in order to determine preciseIy the thickness of the photoresist mask. Further, ion implantation has gained much attention since it was discovered that organic semiconductors might be created by ion beam doping of poiymers.

Wo of the least studied aspects of Lon-implanted and irradiated polymers are the depth distributions of the ions and their energy transfer. The high implantation doses $\left(\phi>5 \times 10^{14}\right.$ atoms $\left./ \mathrm{cm}^{2}\right)$ needed for the usual detection techniques (SIMS, RBS, and AES) can significantly alter the physical and chemical properties of the polymers, and consequently render the polymer useless for range determination purposes. This limitation accounts for the paucity of experimental data on range parameters of ions in photoresists. Nevertheless, in the last years several measurements of ion ranges in polymers have been done. Adesida and Karapiperis ${ }^{1}$ determined the profiles for various light ions in polymethylmethacrylate (PMMA) using the technique of ion beam lithography (IBL). In this technique the polymer is bombarded with ions and subsequently developed in a suitable solvent. The saturated developed depth is interpreted as the mean path length of the implanted ion. In another work Tennant et al. ${ }^{2}$ reported measurements of boron ranges in photoresists using the SIMS technique in an alternative way. They implanted B ions at a fixed energy through a series of polymer thicknesses, measuring the SIMS depth profiles in the underlying Si substrate. In both cases the indirect nature of the used techniques was a limiting factor in the accuracy of the range measurements, and prevented the precise reconstruction of the implanted ion concentration profiles. This detail is important since in a recent work Fink et $a l^{3}$ have shown that light ions ( ${ }^{6} \mathrm{Li}$ and ${ }^{10} \mathrm{~B}$ ) after implantation into epoxy resist and photoresist $\mathrm{AZ} 111$ distribute according to a nonregular shape. As a consequence, further experimental investigations on boron-implantation profiles in photoresist are needed.

In the present work, we have determined the depth distribution of ion-implanted ${ }^{10} \mathrm{Z}$ in $\mathrm{AZ} 111$ photoresist in the $30-150 \mathrm{keV}$ energy range. We used the nuclear reaction analysis (NRA) technique with thermal neutrons, ${ }^{4}$ as it is highly sensitive for ${ }^{10} \mathrm{~B}$ ions and gives direct information on the depth distributions, while producing negligible radiation damage in the sample.

\section{EXPERIMENTAL PROCEDURE AND RESUETS}

Clean silicon wafers were spin coated with $A Z 111$ photoresist of $1 \mu \mathrm{m}$ thickness and baked for $1 \mathrm{~h}$ at $150^{\circ} \mathrm{C}$. Smal pieces of the wafers $\left(\approx 2 \mathrm{~cm}^{2}\right)$ were implanted with fuences of $10^{14}$ atoms $/ \mathrm{cm}^{2}$ at energies of $30,60,90,120$, and $150 \mathrm{keV}$. The implantation was performed at room temperature with low beam current densities $\left(\approx 50 \mathrm{nA} / \mathrm{cm}^{2}\right)$ in order to avoid excessive heating of the samples.

Depth profiles were obtained through the ${ }^{10} \mathrm{~B}(n, \alpha)^{7} \mathrm{~L}$ reaction with thermal neutrons at the high fux reactor of the Institute Laue-Langevin, Grenoble, France. The $\alpha$ particles were detected by an ORTEC silicon surface barrier detector with energy resolution of $14 \mathrm{keV} \mathrm{FWHM}$. For the energy to depth transformation the stopping powers after $Z B L$ were used." All other experimental details can be found in $R$ ef. 4 .

Figures $1(a)-1(\mathrm{c})$ show the ${ }^{10} \mathrm{~B}$ depth distributions after 30,60 , and $150 \mathrm{keV}$ implantation, respectively. Figure shows clearly that between 30 and $60 \mathrm{keV}$ a transition occurs from a regular ion-implantation profle to another one with an additional tail directed towards the surface. This feature is more clearly observed in the depth profile corresponding to $150-\mathrm{keV}$ energy implantation. Then the usual data analysis based on the determination of the four characteristic moments of the particle distribution (projected range $R_{p}$, range straggling $\Delta R_{p}$, skewness $\gamma$, and kurtosis $\beta$ ) should be done with restrictions. This is due to the nonregu- 


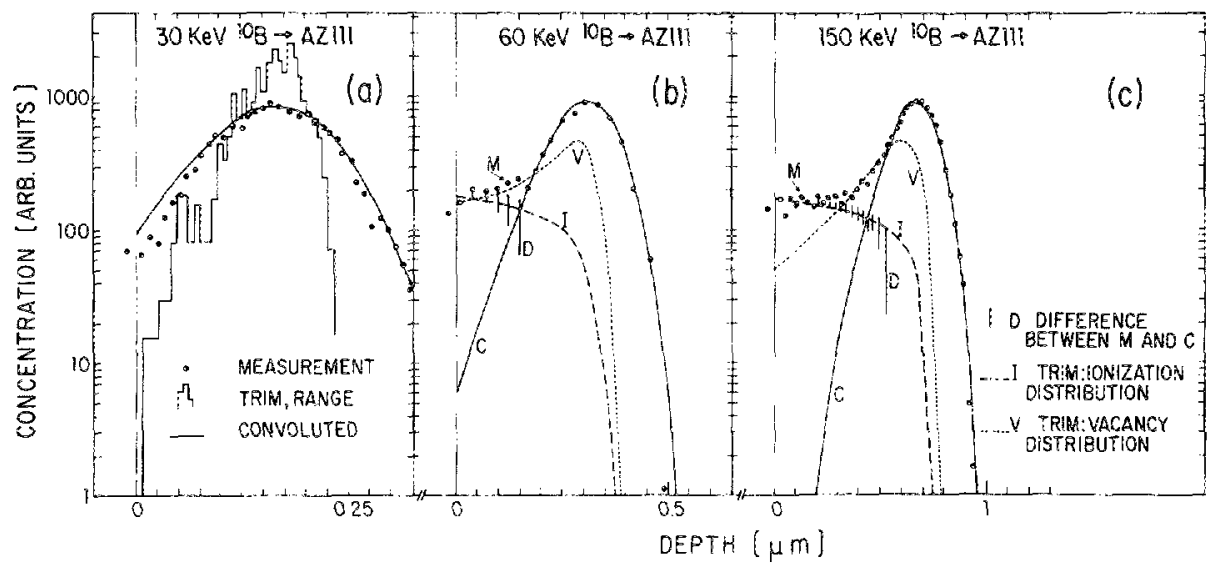

FIG. 1. (a) Experimental depth distribution of ${ }^{10} \mathrm{~B}$ implanted at $30 \mathrm{keV}$ into the AZ111 photoresist (full points). The histogram represents the THIM calculated particle distribution, and the line the TRIM results convoluted with the detector resolution. (b) $60-\mathrm{keV}{ }^{i 0} \mathrm{~B}$ implanted into $A Z 111$. The full points (M) represent the experimental results, the full curve (C) shows the TRIM-convoluted particle distribution, the dotted line (V) the predicted vacancy distribution, and the dashed line (I) the calculated ionization profile. The verticai bars (D) indicate the difference between the experimental results and the TRIM calculated particle distribution. (c) $150-\mathrm{keV}^{10} \mathrm{~B}$ implanted into A2111. The symbols have the same meaning as in (b).

lar character of the ion-implanted profiles. In particular, it is useless to try to determine the higher moments of the particle distributions $\left(\Delta R_{p}, \gamma\right.$, and $\left.\beta\right)$, since comparison with the theory would be meaningless. For evaluation of $R_{p}$ we subtracted the tail distribution from each measured spectrum. This procedure is justified since even in the most extreme case, the tail is only $10 \%$ of the peak height. In addition, we determine directly the position of the most probable range $\hat{R}$ (i.e., the maximum of the distribution). Both values $R_{p}$ and $\widehat{R}$ are quoted in Table $I$ as a function of the energy. As was mentioned above, for the particle distributions corresponding to energy higher than $60 \mathrm{keV}$, determination of the second moment would be meaningless. Therefore, we have characterized the distributions by the full width at halfmaxima (FWHM) which are quoted in Table l.

In order to compare the present results with the latest theoretical predictions, we have used the Monte Carlo code TRIM ${ }^{6}$ with the universal potential developed by Ziegler, Biersack, and Littmark (ZBL) ${ }^{5}$ and an improved electronic stopping power due to Brandt and Kitagawa ${ }^{7}$ as inputs. After convoluting the TRIM results with the detector resolution and the straggling of the ${ }^{4} \mathrm{He}$ particles we have obtained for each implantation energy the corresponding $R_{p}, \hat{R}$, and FWHM which are quoted in Table $I$ and displayed in Fig. 2 , together with the results of the present and previous experiment."

Table $I$ and Fig. 2 show that there is an overall good agreement (within 10\%) between the present experimental results and the theoretical predictions. Figure 2 also shows that the results of Ref. 1 for $B$ implanted into PMMA (which has a composition very similar to the AZ111 photoresist) are larger than the present ones and consequently than the TRM predictions. As was mentioned before, those differences might be attributed to the indirect character of the IBL measuring technique used in Ref. 1 and therefore to the lack of precision in the determination of the ion distribution parameters of the implanted boron. The experimental results of Ref. 2 are not included in the figure since they refer to photoresists with different compositions and densities.

Figure 1(a) shows in addition to the experimental results the calculated and convoluted TRIM particle distributions. The calculated profile follows quite well the experimental points indicating that at $30 \mathrm{keV}$ the ${ }^{10} \mathrm{~B}$ particle distribution is regular. This is not the situation for higher energies. To get some insight into the origin of the nonregularity of the ion distributions we have plotted in Figs. $1(b)$ and 1(c) the TRIM convoluted particle (full line), vacancy (dotted line), and ionization (dashed line) distributions. They were arbitrarily normalized to the experimental resuits. Figure 1 (c) suggests that the tail follows the ionization profile as calculated by the TRIM. For further clarification we subtracted the corresponding convoluted particle distribution (as predicted by TRIM) from each experimental profile. The results are depicted by vertical bars, the lengths indicating the uncertainty introduced by this operation. It is clearly seen in both figures that the bars (which are identical to the points where there is ro TRMM particle contribution) follow very closely the TRIM calculated ionization profile.

TABLE 1. Experimental and TRIM calculated range parameters for ${ }^{10} \mathrm{~B}$ implanted into the AZ1 11 polymer at various energies. Typical errors around $4 \%$.

\begin{tabular}{|c|c|c|c|c|c|c|c|}
\hline \multirow[b]{2}{*}{$\begin{array}{l}\text { Energy } \\
(\mathrm{keV})\end{array}$} & \multicolumn{3}{|c|}{ Experimental } & \multicolumn{4}{|c|}{ TRIM predictions } \\
\hline & $\begin{array}{c}\hat{R} \\
(\mathrm{~nm})\end{array}$ & $\begin{array}{c}R_{p} \\
(\mathrm{~nm})\end{array}$ & $\begin{array}{c}\text { FWHM } \\
(\mathrm{nm})\end{array}$ & $\begin{array}{c}\hat{R} \\
(\mathrm{~nm})\end{array}$ & $\begin{array}{c}R_{p} \\
(\mathrm{~nm})\end{array}$ & $\begin{array}{l}\text { FWHM } \\
(\mathrm{nm})\end{array}$ & $\begin{array}{c}\text { FWHM } \\
\text { convoluted } \\
\text { (nm) }\end{array}$ \\
\hline 30 & 162 & 154 & 155 & 160 & 153 & 60 & 165 \\
\hline 60 & 275 & 270 & 165 & 300 & 290 & 85 & 170 \\
\hline 90 & 378 & 390 & 175 & 430 & 415 & 85 & 170 \\
\hline 120 & 552 & 520 & 210 & 565 & 528 & 140 & 195 \\
\hline 150 & 670 & 610 & 240 & 690 & 622 & 170 & 240 \\
\hline
\end{tabular}




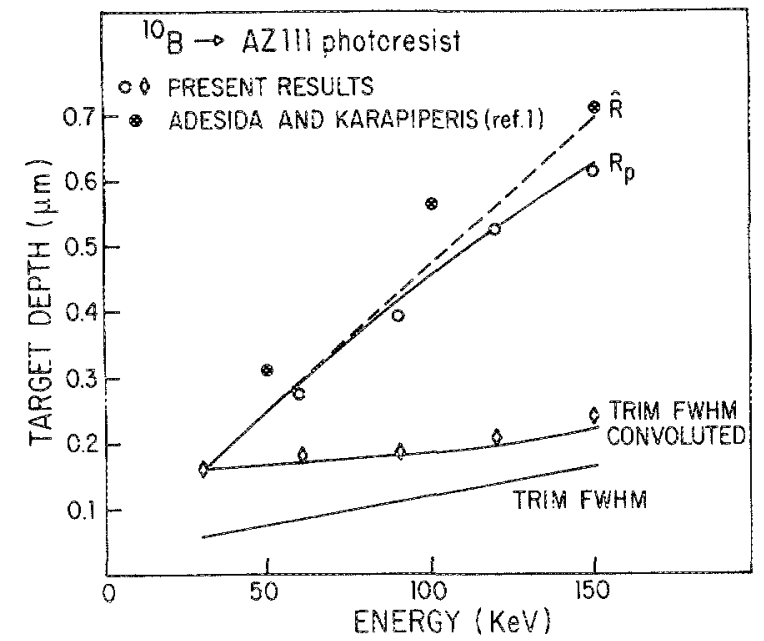

FIG. 2. Experimental and theoretical range profile paraneters as a function of the energy for ${ }^{10} B$ implanted into the photoresist $A Z 111$. The lines represent the TRIM theoretical predictions for $\hat{R}$ (dashed line), $R_{p}$ (upper full

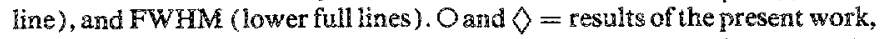
= results of Adesida and Karapiperis. Note that at $30 \mathrm{keV}$, the $R_{p}$ and the FWHM are identical.

This feature is also observed for the 90 and $120 \mathrm{keV}$ cases which are not displayed in Fig. 1.

\section{DISCUSSION AND CONCLUSION}

From the results of the present work several conciusions can be drawn. First, at about $60 \mathrm{keV}$ the boron-implantation profle suffers a change to a nonregular shape. At this energy around $10 \%$ of the implanted ions distribute according to the ionization profile. In addition, there are indications that with increasing implantation energy this number increases, reaching a value of about $15 \%$ at $150 \mathrm{keV}$. This number is in agreement with the recent findings of Fink et al. ${ }^{8}$ By implanting ${ }^{10} \mathrm{~B}$ at a fixed energy $(200 \mathrm{keV})$ in different kinds of polymers the authors of Ref. 8 found that in all cases typical1y $90 \%$ of the implanted ions follow the regular profile as predicted by the TRIM, while the remaining $10 \%$ follow the ionization profile.

It should be also pointed out that the change in shape observed in the present work for the $B$ profile occurs at an energy where the electronic stopping power $S_{e}$ is higher by a factor of 5 than the nuclear one $S_{n}$, t.e.s $S_{k} \approx 5 S_{n}$. This should be compared to a previous result for fluorine also implanted in the AZ111 photoresist ${ }^{9}$ where the profile changes from a nearly regular shape to a nonregular one at an implantation energy for which $S_{e} \approx 2 S_{n}$.

In order to explain the transition from the regular range distribution towards the ionization profile, one has to assume a certain mobility of the implanted light ions. The diffusing lons may be trapped by free radicals which are created by electronic energy transfer processes efficiently enough only for $S_{e} \geqslant S_{n}$, so that the ion depth distribution approaches the shape of the ionization distribution. Since this is still a very tentative explanation, more experimental work should be done.

Second, it should be pointed out that despite the nonregularity of the implanted-ion distributions one can still characterize them by some range parameters like $R_{p}, \hat{R}$, and FWHM. The TRM predictions with the ZBL stopping power reproduce quite well the above parameters, and therefore at least for $B$ in $A Z 111$ photoresist they can be regarded as reliable.

Finally, it should be stressed that a direct technique like NRA with thermal neutrons used in the present work, yields more information about the profiles of implanted ions than the indirect techniques of Refs. 1 and 2. In fact, in previous works there were no hints about the nonregularity of the implanted boron profiles in polymers while in the present work this feature clearly shows up.

\section{ACKNOWLEOGMENTS}

This work was supported by the Institute Laue-Langevin (ILL) Grenoble. One of the authors (M.B.) acknowledges the kind hospitality at the Hahn-Meitner-Institute, Berlin.

1. Adesida and L. Karapiperis, J. Appl. Phys, 56, 1801 (1984) and references therein.

${ }^{2}$ D. M. Tennant, A. H. Dayem, R. E. Howard, and E. H. Westerwick, J. Vac. Sci. Technol. B 3, 458 (1985).

${ }^{3}$ D. Fink, J. P. Biersack, J. T. Chen, M. Städele, K. Tjan, M. Behar, C. A. Olivieri, and F. C. Zawislak, J. Agpl. Phys. 58, 663 (1985).

4. P. Biersack, D. Fink, R. Henkelmann, and K. Mülier, Nucl. Instrum. Methods 149, $93(1978)$.

${ }^{5}$ J. F. Ziegler, J. P. Biersack, and U. Littmark, in The Stopping and Range of Ions in Solids, edited J. F. Ziegler (Pergamon, New York, 1985), Vol. 1.

${ }^{5}$ J. P. Biersack and L. G. Haggmark, Nucl. Instrum. Methods 1\%4, 257 (1980).

7W. Brand and M. Kitagawa, Phys. Rev. B 25, 5631 (1982).

${ }^{B}$ D. Fink, M. Müller, U. Sttettner, M. Behar, P. F. R. Fichtner, F. C. Zawislak, and S. Koul (to be published).

${ }^{9}$ R. B. Guimarães, M. Behar, R. P. Livi, J. P. de Souza, L. Amară, F. C. Zawiskak, D. Fink, and J. P. Biersack, Nucl. Instrum. Methods B 19/20, $882(1987)$. 